\title{
HYPOXIA AND HEARING - WHAT DO WE REALLY KNOW?
}

\author{
Simone van der Giet ${ }^{1(\mathrm{~A}-\mathrm{G})}$, Paul Jansing ${ }^{2,3(\mathrm{D}-\mathrm{F})}$, Thomas Küpper ${ }^{1,4(\mathrm{~A}-\mathrm{G})}$ \\ ${ }^{1}$ Institute and Outpatient Clinic of Occupational and Social Medicine, RWTH Aachen University, Aachen, Germany \\ ${ }^{2}$ State Institute for Occupational Health and Safety of North-Rhine Westphalia, Düsseldorf, Germany \\ ${ }^{3}$ Institute of Occupational Medicine, Heinrich-Heine-University Düsseldorf, Germany \\ ${ }^{4}$ Medical Commission of the Union Internationale des Associations d'Alpinisme (UIAA MedCom)
}

\begin{abstract}
Due to the fact, that the hearing process is an energy-depended and consumptive process, some changes happen, when going to high altitude where the oxygen partial pressure decreases. The oxygen is provided to the inner ear by diffusion over a relatively large distance. Because the amount of material decreases with the square of the distance high noise levels at altitude or in isobaric hypoxia must be assumed as a stress situation for the inner ear. There are only a few data about a temporary or permanent threshold shift in hypoxia or an increased sensitivity of the ear for occupational noise disease in such conditions. The hearing loss due to hypoxia at altitude or in isobaric hypoxia is a central-nervous hypoxic dysfunction and not rarely a sign of a beginning HACE. We review the actual knowledge about this topic.
\end{abstract}

Key words: hypoxia, hearing, noise protection, alpine rescue, isobaric hypoxia

\section{Introduction}

About 300.000 persons per year go to high altitude worldwide, either to work, to travel or for helicopter operations in rescue or military missions. There is an additional group where nobody knows the number of persons exposed, but which is increasing significantly since some years: people working in isobaric hypoxia (server rooms, special rooms with hypoxia for fire protection, storage rooms for fruits and vegetables, and many others). Several tasks are a combined exposure with noise. We summarize the actual knowledge about the problem and summarize the consequences for further research.

\section{Physiology of hearing in hypoxia}

In contrast to normoxia it must be assumed for any kind of hypoxia that there will be a stress situation for the inner ear when the receptor cells are exposed to noise since hearing is an energy-consuming process with more energy needed at high noise levels and the reservoir of energy-rich phosphates (ATP) of the cells of the stria vascularis is limited [1]. But noise exposure reduces the blood flow in the stria vascularis even more and therefore a more restricted energy pool must be expected $[2,3]$. This is supported by the finding that oxygen partial pressure decreases in the perilymphatic liquid [3]. These effects are correlated to the noise level and continue after the noise exposure for about one hour [3].

An energetic exhaustion of the hairy cells is assumed as the physiological mechanism which causes the temporary threshold shift (TTS) as the first step towards a permanent hearing deficiency (permanent threshold shift, PTS). When the noise exposure continues and the ear doesn't get the chance to recover adequately from the energy deficit a PTS will be the consequence with a degeneration of the hairy cells and (later) the associated nerve fibres will occur.

With the situation described above it may be expected that a combined exposure of noise and hypoxia the TTS (and later the PTS) arises earlier since the energetic deficiency occurs earlier in hypoxia. Indeed, there are results obtained in animal which prove a TTS [4]. Limited data indicate a similar effect in humans at conditions which correlate to about $4500 \mathrm{~m}$ [5]. Animals which were acclimatized to high altitude showed significant less TTS than those without acclimatization [6]. As for the other organs, several processes like increased blood flow, hemoconcentration, ventilatory acclimatization, and others, all of which are commonly summarized as "acclimatization", increase the oxygen supply of the inner ear. This may explain the effect of acclimatization on TTS described by Berndt at al. [7].

Such a hearing reduction has been already described by Farland et al. in 1938 from people in the high Andes at altitudes above $5930 \mathrm{~m} \mathrm{[8].} \mathrm{On} \mathrm{the} \mathrm{other}$ hand there are others who published that the auditory threshold remained unchanged in hypoxic conditions [9] or that an increase of the haematocrit to $51 \%-58 \%$ does not influence the hearing potentials and there is a stability in the otoacoustic emissions although the cochlear blood flow decreases to $40 \%$ compared 
to normoxia or sea level MSL in the animal model $[10,11]$. First results of the Aachen Dental and Medical Expedition 2011 (ADEMED 2011, www.ademed.de) also indicate a higher risk for TTS at high altitude and a significant protecting effect of acclimatization [12].

\section{Consequences for noise protection and further research}

Limited data and physiological considerations suggest that there is an increased risk for hearing loss due to hypoxia and high altitude, but there is still a lack of detailed results which prove the theory. This should be investigated by a collective with similar - ideally the same - altitude profile from sea level up to at least $5,000 \mathrm{~m}$ and then down to sea level again to check for acclimatization effects. Other effects of hypoxia or altitude like the aggravation for the ability to locate sound origins [13] or that the risk for acoustic hallucinations increases [7] are more technical problems of such investigations and should be avoided easily by experimental designs like objective measurements of the TTS by automated systems which give the stimuli of the several frequencies randomized to the probands.

Another interesting topic is the possibility of a pharmacological protection of the ears when exposed to noise in hypoxic environment. Experiments with animals with the selective glutamate antagonist MK 801 showed that this is principally possible [14].

The criteria for amblyacusia caused by noise exposure as an occupational disease are developed in normoxia [15-18]. With the considerations mentioned above these criteria are should not be valid for hypobaric (altitude) or isobaric hypoxia. In consequence, assumed that the considerations mentioned above are true, there are several persons whose hearing loss is not accepted as noise-induced, although it is a noiserelated damage and only criteria which do not take the special conditions of the exposure into account were used. This needs more data to establish criteria for noise disease in hypoxic conditions as well as for preventive recommendations for the persons exposed. Until such data are available anybody - employees, patients, bystanders, and others - should take special care for their ears when exposed to noise in any kind of hypoxic environment.

\section{References}

1. Munoz DJ, Kendrick IS, Rassam M, et al. Vesicular storage of adenosine triphosphate in the guinea-pig cochlear lateral wall and concentrations of ATP in the endolymph during sound exposure and hypoxia. Acta Otolaryngol 2001; 121(1): 10-5.
2. Attanasio G, Buongiorno G, Piccoli F, et al. Laser Doppler measurement of cochlear blood flow changes during conditioning noise exposure. Acta Otolaryngol 2001; 121(4): 465-9.

3. Lamm K, Arnold W. Noise-induced cochlear hypoxia is intensity dependent, correlates with hearing loss and precedes reduction of cochlear blood flow. Audiol Neurootol 1996; 1(3): 148-60.

4. Attias J, Sohmer H, Gold S, et al. Noise and hypoxia induced temporary threshold shifts in rats studied by ABR. Hear Res 1990; 45(3): 247-52.

5. Fowler B, Grant A. Hearing thresholds under acute hypoxia and relationship to slowing in the auditory modality. Aviat Space Environ Med 2000; 71(9 Pt 1): 946-9.

6. Berndt $\mathrm{H}, \mathrm{Kranz} \mathrm{D}$, Wagner $\mathrm{H}$, et al. [Hair cell noise damage after improved hypoxia tolerance (author's transl)]. Laryngol Rhinol Otol (Stuttg) 1978; 57(6): 520-3.

7. Mees K, Suckfull M. [Cochlear and vestibular risk at high altitude]. Laryngorhinootologie 2002; 81(7): 465-8.

8. McFarland RA. Psycho-pysiological Studies at High Altitude in the Andes. J Comp Psychology 1938; 23(2): 147-88.

9. Burkett PR, Perrin WF. Hypoxia and auditory thresholds. Aviat Space Environ Med 1976; 47: 649-51.

10. Lindan D, Yedgar S, Aronson H, et al. Influence of experimentally elevated blood viscosity on the auditory nerve-brainstem evoked response and Threshold. Hear Res 1992; 62(1): 57-62.

11. Suckfüll M, Mees K. Haemoconcentration as a possible pathogenetic factor of sudden hearing loss. Eur Arch Otorhinolaryngol 1998; 255: 281-4.

12. van der Giet S, Bartz N, Bertsch D, et al. The risk for hearing loss and temporary threshold shift at altitude. (in preparation).

13. Rosenberg ME, Pollard AJ. Altitude-dependent changes of directional hearing in mountaineers. Br J Sports Med 1992; 26(3): 161-5.

14. Selvadurai DK, Etheridge S, Jones P, et al. Pharmacological protection of auditory function against noise and hypoxia with MK 801. Clin Otolaryngol 2000; 25(6): 570-6.

15. N.N. Occupational noise exposure. Code of Federal Regulations 1983; 29(1910.95): 176-93.

16. N.N. Noise at work regulations., London: HMSO, 1989.

17. N.N. Lärm (G20) [Noise-induced hearing damage]. In: Berufsgenossenschaften Hdg, Editor. Arbeitsmedizinische Vorsorge, Stuttgart: Gentner Verlag, 1994: 281-94.

18. N.N. Directive 2003/10/EC of the European Parliament and of the Council of 6 February 2003 on the minimum health and safety requirements regarding the exposure of workers on the risk arizing from physical agents (noise) (Seventeenth individual Directive within the meaning of Article 16(1) of Directive 89/391/EEC). Official Journal European Union 2003; L42: 38-44

Received: January 04, 2012

Accepted: June 04, 2012

Published: June 29, 2012

Address for correspondence:

Simone van der Giet, $\mathrm{MD}, \mathrm{PhD}$

Institute of Occupational and Social Medicine

RWTH Aachen University

Pauwelstr. 30

D-52074 Aachen

Deutschland

Email: fetzenfischchen@gmx.de

Paul Jansing: jansing@uni-duesseldorf.de Thomas Küpper: tkuepper@ukaachen.de

\begin{tabular}{llll}
\hline Authors' contribution & B - Data Collection & D - Data Interpretation & F - Literature Search \\
A - Study Design & C - Statistical Analysis & E - Manuscript Preparation & G - Funds Collection
\end{tabular}

